\title{
Non-Invasive Assessment of Spatiotemporal Organization of Ventricular Fibrillation Through Principal Component Analysis
}

\author{
Marianna Meo ${ }^{1,2,3}$, Mark Potse ${ }^{1,4,5}$, Stéphane Puyo ${ }^{1,6}$, Laura Bear ${ }^{1,2,3}$, Mélèze Hocini ${ }^{1,2,3,6}$, Michel \\ Haïssaguerre, Rémi Dubois ${ }^{1,2,3}$ \\ ${ }^{1}$ IHU LIRYC, Electrophysiology and Heart Modeling Institute, Foundation Bordeaux University, \\ Pessac-Bordeaux, France \\ ${ }^{2}$ Univ. Bordeaux, CRCTB, U1045, Bordeaux, France \\ ${ }^{3}$ INSERM, CRCTB, U1045, Bordeaux, France \\ ${ }^{4}$ CARMEN Research Team, INRIA, Talence, France \\ ${ }^{5}$ Univ. Bordeaux, IMB, UMR 5251, Talence, France \\ ${ }^{6}$ Bordeaux University Hospital (CHU), Electrophysiology and Ablation Unit, Pessac, France
}

\begin{abstract}
Introduction Ventricular fibrillation (VF) is the main cause of sudden cardiac death, but we lack tools to predict the evolution of its complexity. This study proposes novel $V F$ complexity markers obtained by principal component analysis (PCA) of body surface potential maps (BSPMs). Methods BSPMs were divided in 0.5-s segments, each projected on the $3 D$ PCA subspace determined in the previous frame. Reconstruction error $\epsilon$ was expressed in terms of norm $d_{\epsilon}$ and angle cosine $\cos \left(\alpha_{\epsilon}\right)$, and the nondipolar component index (NDI) quantified the energy of the first 3 PCA eigenvalues. These markers quantified changes in complexity between the beginning and the end of $24 \mathrm{VF}$ episodes and 5 control sinus rhythm (SR) recordings. They were also tested on 6 BSPMs from a torso-tank model during and after ex-vivo pig heart perfusion. Differences between in-silico BSPM right and left leads were then verified in 4 human VF simulations, with a reentrant source in the right ventricle. Results Higher NDI and $d_{\boldsymbol{\epsilon}}$ and lower $\cos \left(\alpha_{\epsilon}\right)$ denoted higher complexity at the end of $V F$ $(p<0.0001)$. No changes occurred in SR $(p>0.05)$. The indices also underlined higher disorganization in exvivo non-perfused VF $(p<0.0001)$. A similar trend was observed in the right in-silico BSPM leads $(p<0.05)$. Conclusions PCA can non-invasively quantify changes in $V F$ complexity.
\end{abstract}

\section{Introduction}

Ventricular fibrillation (VF) is the most serious heart rhythm disturbance and the main cause of sudden cardiac death [1]. However, there is still a lack of quantitative tools to evaluate the degree of myocardial electrical organization during VF and predict its evolution. Most of the data used to test hypotheses regarding VF mechanisms come from animal models and computer simulations [2]. VF appears to be triggered and maintained by high frequency reentrant sources, with the spatiotemporal organization quantified through optical mapping of the heart surface in terms of phase locations and singularities [3]. To date, very few experimental studies on human VF have been reported [4], and clinical applications of non-invasive cardiac electrical mapping are still limited to a few centers [5]. VF onset was predicted by some complexity measures in electrocardiograms (ECGs) from animal models [6] and humans [7], but intra-episode organization was not evaluated. In [8], atrial activity organization during atrial fibrillation (AF) was assessed by principal component analysis (PCA) of body surface potential maps (BSPMs). In [9], PCA features from multilead ECGs proved to be predictive of $\mathrm{AF}$ ablation outcome. In both studies, the distance between the input signal and its reduced-rank PCA approximation was used to measure AF organization. In this paper, we move from this research to develop a novel PCA-based approach and evaluate changes in VF complexity in BSPMs. Our method was applied to clinical BSPMs acquired during VF and compared to recordings in normal sinus rhythm (SR). Its ability to detect changes in VF organization was also tested on torso potential recordings from a Langendorff system. Finally, it was validated on in-silico body surface signals generated by a VF human heart model. 


\section{Methods}

\subsection{Clinical VF database}

BSPMs from 24 patients ( 22 male, $50 \pm 22$ years old) were acquired with a 252-electrode vest (ECVUE, Cardioinsight Technologies, Cleveland, OH, USA) during episodes of spontaneous or induced VF (duration $19.8 \pm$ $6.5 \mathrm{~s}$ ). An example is shown in Figure 1. VF was accompanied by structural heart diseases (SHD) in 7 patients ( 5 with ischemic VF, 2 with microvascular coronary dysfunction). VF was associated with Brugada and/or early repolarization syndrome in 12 non-SHD patients, and 5 were diagnosed with idiopathic VF. Five BSPMs from 3 male subjects ( $38 \pm 13$ years old) in normal sinus rhythm (SR) were used as a control group.

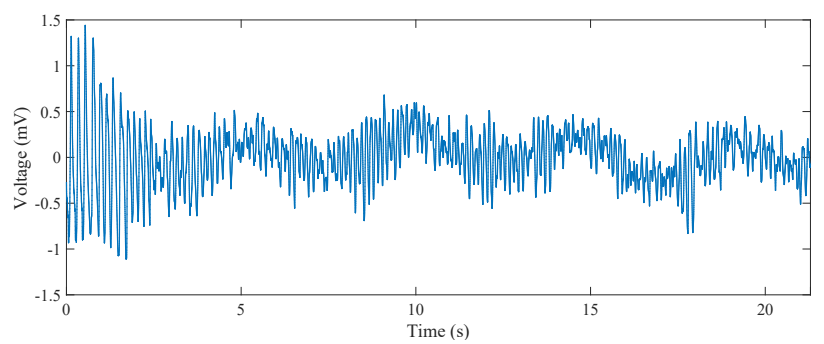

Figure 1. A BSPM recording (lead I) from a VF patient.

\subsection{BSPMs from ex-vivo experiments}

Ex-vivo data were collected from 6 Langendorffperfused pig hearts with standard Tyrode's solution in a human-shaped torso tank. A flexible 108-electrode sock was placed over the epicardium and a pacing lead on the right ventricle (RV) apex [10]. Tank and sock potentials were acquired at 128 electrodes at $2 \mathrm{kHz}$ (BioSemi, the Netherlands), referenced to a Wilson's central terminal. Constant current stimuli were injected with shortening pulse durations starting at $1 \mathrm{~ms}$ to induce VF. In two cases rapid pacing induced ventricular tachycardia (VT), which later degenerated to $\mathrm{VF}$, and in four cases there was a straightforward transition from SR to VF (duration: $213 \pm 143 \mathrm{~s}$ ). Perfusion was stopped after 10 minutes (duration: $537 \pm 414 \mathrm{~s}$ ) to mimic conditions for realistic VF.

\subsection{BSPMs from a human heart model}

VF complexity markers were also tested on a bidomain finite-difference torso model (1-mm resolution) combined with a monodomain reaction-diffusion heart model $(0.2-$ mm resolution) [12]. We used an MRI-based patientspecific $3 D$ heart-torso model with anisotropic conduction properties in the heart derived from rule-based fiber orientation. Transmembrane currents were computed with a Ten
Tusscher-Panfilov 2006 (TP06) human ventricular tissue model [11] to generate a 252-electrode BSPM (Figure 2, right). VF-like excitation patterns were reproduced for $10 \mathrm{~s}$ using the standard model tuning settings. Electrical instability was also simulated by increasing action potential duration restitution slope and fast sodium current dynamics recovery speed [11], thus generating reentrant waves and spiral breakups, with the core located in the RV (Figure 2, left), whereas LV was overall passive. Consequently, propagation pattern complexity was separately assessed by our method in each side of the heart after splitting the BSPM leads in 2 distinct sets in 4 simulations.
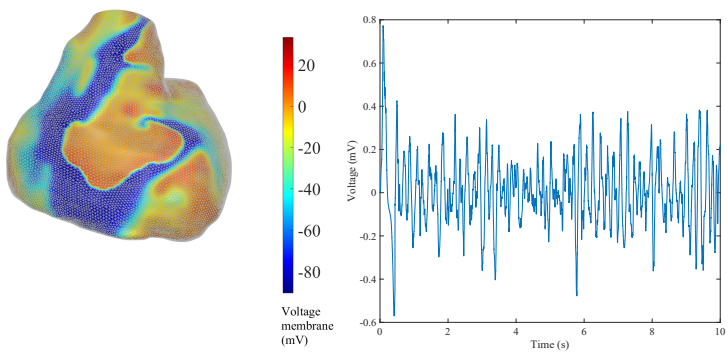

Figure 2. Voltage membrane distribution (left) and body surface potential (lead I, right) from a VF simulation.

\subsection{BSPM data format and preprocessing}

Prior to PCA, BSPMs were mean-centered and arranged as an $L \times N$ matrix $\mathbf{Y}=[\mathbf{y}(1) \ldots \mathbf{y}(N)] \in \Re^{L \times N}$, where $L$ is the number of leads, and $N$ the number of samples. We examined $L=252$ leads in the in-silico and clinical BSPMs, $L=128$ leads in the ex-vivo BSPMs. In real data, electrodes with excessive noise level were discarded after visual inspection of the signals, thus in certain cases less than $L$ electrodes were retained. Furthermore, a wavelet bandpass filter was applied to tank data in the frequency band $[3-150] \mathrm{Hz}$ to remove noise due to baseline wander and high frequency components.

\subsection{PCA transformation of BSPM data}

We investigated whether the degree of temporal repetitiveness of PCA features could measure VF pattern complexity. To this end, BSPMs were divided in $N_{s}=0.5$-s segments, and in each of them singular value decomposition of the input data $\mathbf{Y}^{(s)}$ was applied as in $[8,9]$ :

$$
\mathbf{Y}^{(s)}=\mathbf{U S V}^{T}
$$

where $\mathbf{U}$ and $\mathbf{V}$ contain the left and right singular vectors of $\mathbf{Y}^{(s)}$, respectively, and the diagonal matrix $\mathbf{S}$ contains the singular values $\sigma_{\ell}, \ell=1, \ldots, L$, each associated with the principal components (PCs), which are mutually uncorrelated and linked with the observations through the re- 
lation $\mathbf{Y}^{(s)}=\mathbf{M}^{(s)} \mathbf{X}^{(s)}$, where $\left.\mathbf{M}^{(s)}=\mathbf{U S} / \sqrt{(} N_{s}\right)$ is the PCA mixing matrix. Since the heart can be represented as an electric dipole [13] and most of the body surface potential energy can be adequately described by the first 3 PCs [14], we retained a 3D subspace $\mathbf{M}_{3}^{(s)}$ as spanned by the first 3 highest-variance columns of $\mathbf{M}^{(s)}$.

\subsection{BSPM complexity feature extraction}

In order to test the ability of PCA components to be retrieved along the BSPM, each signal $\mathbf{Y}^{(s+1)}$ in the $(s+1)$ th segment was projected onto the subspace determined in the preceding frame $(s)$ :

$$
\hat{\mathbf{Y}}^{(s+1)}=\mathbf{M}_{3}^{(s)}\left(\mathbf{M}_{3}^{(s)}\right)^{\#} \mathbf{Y}^{(s+1)}
$$

where $(\cdot)^{\#}$ is the Moore-Penrose pseudoinverse operator. The instantaneous multilead error $\epsilon$ between the input signal $\mathbf{y}^{(s+1)}$ and its PCA approximation $\hat{\mathbf{y}}^{(s+1)}$ was computed. From a geometric point of view, these two terms can be regarded as vectors (with as many components as the number of BSPM leads examined), whose degree of similarity can be evaluated in terms of magnitude and direction. To this end, we computed the normalized amplitude norm of the PCA reconstruction error as:

$$
d_{\boldsymbol{\epsilon}}=\frac{\left\|\hat{\mathbf{y}}^{(s+1)}(t)-\mathbf{y}^{(s+1)}(t)\right\|}{\left\|\mathbf{y}^{(s+1)}(t)\right\|} .
$$

Amplitude normalization was performed to avoid bias due to different signal voltage scales, so that $d_{\boldsymbol{\epsilon}}$ values will depend on PCA approximation accuracy only. Cosine similarity between the two vectors was also computed as:

$$
\cos \left(\alpha_{\boldsymbol{\epsilon}}\right)=\frac{\left\langle\hat{\mathbf{y}}^{(s+1)}(t), \mathbf{y}^{(s+1)}(t)\right\rangle}{\left\|\hat{\mathbf{y}}^{(s+1)}(t)\right\|\left\|\mathbf{y}^{(s+1)}(t)\right\|},
$$

so that values close to 1 will depict more similar and correlated signal patterns. Finally, the nondipolar component index (NDI) was determined as a function of energy retained by the PCA eigenvalues $\sigma_{\ell}, \ell=4, \ldots, L$ outside the projection subspace described by $\mathbf{M}_{3}^{(s)}$ columns.

$$
\mathrm{NDI}=1-\frac{\sum_{\ell=1}^{3} \sigma_{\ell}}{\sum_{\ell=1}^{\mathrm{L}} \sigma_{\ell}}
$$

Lower NDI values indicate that BSPM content can be well described by the subspace selected, whereas higher values point out the need for a higher number of PCs to obtain sufficient estimation accuracy. The parameter was computed in $N_{s}$-s sliding windows at a fixed step $q=0.1 \mathrm{~s}$.

\subsection{Statistical analysis}

Parameters' distribution was checked using a Lilliefors test. For normally distributed data, intergroup differences were verified by an unpaired Student's t-test. In the other cases, a Wilcoxon's rank sum test was applied. Significance was taken for $p$-value $\leq 0.05$. Temporal changes in VF complexity were verified in clinical data by comparing features averaged over 4-s windows at the beginning ("Start-VF") and at the end ("End-VF") of the output parameter series. The same analysis was performed in SR ("Start-SR" vs "End-SR"). The choice of this window size is justified by the need to include at least 2-3 cardiac cycles for pattern characterization during SR. PCA indices determined in ex-vivo data and averaged in $N_{s}$-s consecutive frames during ("Perfused VF") and after ("Non-perfused VF") heart perfusion were also examined. Finally, PCA features from the "Right" in-silico BSPM leads were compared to those obtained from the "Left".

\section{Results}

An example of the output temporal series of each of the proposed VF complexity markers is shown in Figure 3.

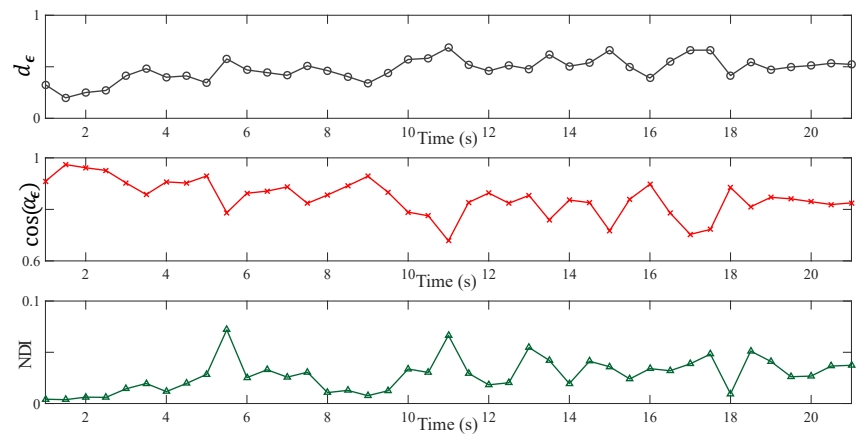

Figure 3. Time series of the PCA features averaged in consecutive 0.5-s frame for the BSPM example in Figure 1.

The distribution of PCA features was displayed through Tukey's box plots and significant comparisons marked with $\left({ }^{*}\right)$ in Figure 4. A significant increase in complexity at the end of the VF episode was quantified by higher NDI and $d_{\boldsymbol{\epsilon}}$ and lower $\cos \left(\alpha_{\boldsymbol{\epsilon}}\right)$ values $(p<0.0001$, Figure 4A). By contrast, no significant changes occurred during SR $(p>0.05)$. A similar trend was observed in the exvivo data before and after stopping perfusion $(p<0.0001$, Figure 4B). Indeed, either the transition from VT to VF or the degeneration of simple VF into more disorganized forms yielded more variable and unpredictable signal patterns. Finally, the same analysis was applied to the synthetic BSPMs in each side of our torso model. The spatial distribution of PCA parameters appears inhomogeneous, and higher VF complexity was significantly quantified by higher $d_{\boldsymbol{\epsilon}}(p=0.01)$ and NDI $(p<0.0001)$ and lower $\cos \left(\alpha_{\boldsymbol{\epsilon}}\right)(p=0.007)$ values on the right side of the heart (Figure 4C), where the VF source was mainly located. 
A.

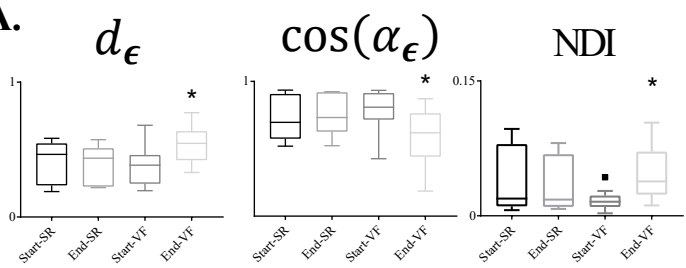

B.

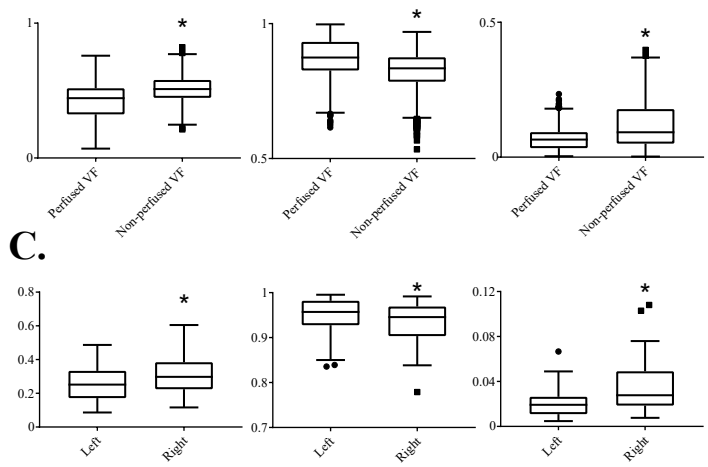

Figure 4. Box plot of the BSPM complexity markers: A. From SR and VF clinical data. B. From ex-vivo data in perfused and non-perfused VF. C. From in-silico BSPM right and left leads.

\section{Discussion and conclusions}

Our study put forward a non-invasive methodology to assess changes in spatiotemporal organization of $\mathrm{VF}$ through suitable features determined by PCA of multilead BSPMs. All the markers could significantly capture and quantify the increase in VF episode complexity which could be visually observed in clinical data. They were also able to characterize the stability of BSPM signals during normal SR, with a low false alarm rate. More disorganized and chaotic patterns were also correctly depicted by our indices in experimental data. Indeed, they effectively detected not only sharp rhythm transitions (e.g., VT/VF), but also alterations due to perfusion interruption, which was responsible for the progressive cardiac tissue degeneration and ischemia onset. VF pattern complexity characterization was also effectively achieved on synthetic BSPMs, with PCA-based parameters indicating higher complexity in the right BSPM leads of our torso model. These results were consistent with the membrane potential distribution at the basis of the generation of a reentrant pattern in the RV. Our findings demonstrate the ability of PCA to noninvasively quantify changes in signal complexity during VF. Future works include the validation of these markers with intracardiac recordings. However, the choice of the proper feature (e.g., entropy, dominant frequency, phase mapping ...) is still an open issue. In-silico results may be also corroborated by a comparison with epicardial poten- tial phase mapping.

\section{Acknowledgements}

This work was supported by the National Research Agency (ANR-10-IAHU04-LIRYC) and the European Research Council (SYMPHONY). It was granted access to the HPC resources of IDRIS under the allocation x2016037379 made by GENCI.

\section{References}

[1] Priori SG, Blomström-Lundqvist C, Mazzanti A et al. 2015 ESC Guidelines for the management of patients with ventricular arrhythmias and the prevention of sudden cardiac death. Eur Heart J 2016;32:2793-2867.

[2] Park SA and Gray RA. Optical mapping of ventricular fibrillation dynamics. Adv Exp Med Biol 2015; 859:313-342.

[3] Umapathy K, Nair K, Masse S et al. Phase mapping of human fibrillation. Circ Arrhythm Electrophysiol 2010; 3:105-114.

[4] Nanthakumar K, Jalife J, Massé et al. Optical mapping of Langendorff-perfused human hearts: establishing a model for the study of ventricular fibrillation in humans. Am J Physiol Heart Circ Physiol 2007; 293(1):H875-H880.

[5] Haïssaguerre M, Hocini M, Nademanee K et al. Driver sources underly the initial stage of ventricular fibrillation. N Engl J Med 2017; under review.

[6] Yua D, Smalla M, Harrisona RG et al. Measuring temporal complexity of ventricular fibrillation. Phys Lett 2000; 265(1): 68-75.

[7] Zhang X, Zhu Y, Thakor N and Wang Z. Detecting ventricular tachycardia and fibrillation by complexity measure. IEEE Trans Biom Eng. 1999; 46(5):548-555.

[8] Bonizzi P, Guillem M de L, Climent A M et al. Noninvasive assessment of the complexity and stationarity of the atrial wavefront patterns during atrial fibrillation. IEEE Trans Biomed Eng. 2010; 57(9): 2147-2159.

[9] Meo M, Zarzoso V, Meste O et al. Catheter ablation outcome prediction in persistent atrial fibrillation using weighted principal component analysis. Biom Signal Process Control 2013. 8(6): 958968.

[10] Bear L, Huntjens P, Coronel R et al. Detection of Incomplete Left Bundle Branch Block by Non-invasive Electrocardiographic Imaging. In. Computing in Cardiology (CinC) 2016, 43: Vancouver, Canada.

[11] Ten Tusscher KHWJ and Panfilov AV. Alternans and spiral breakup in a human ventricular tissue model. Am J Physiol Heart Circ Physiol 2006. 291: H1088-H1100.

[12] Potse M, Dubé B, Richer J et al. A comparison of monodomain and bidomain reaction-diffusion models for action potential propagation in the human heart. IEEE Trans Biomed Eng. 2006. 53(12): 2425-2435.

[13] Holt JH, Barnard CL, Lynn MS and Svendsen P. A Study of the Human Heart as a Multiple Dipole Electrical Source I. Normal Adult Male Subjects. Circ. 1969; 40: 687-696.

[14] Lux RL, Evans AK, Burgess MJ et al. Redundancy reduction for improved display and analysis of body surface potential maps. I. Spatial compression. Circ Res 1981;49:186-196.

Address for correspondence:

Marianna Meo

IHU LIRYC Av. Hâut Lèvéque 33604 Pessac-Bordeaux, France marianna.meo@ihu-liryc.fr 